\title{
Silencing of Host Basal Defense Response-Related Gene Expression Increases Susceptibility of Nicotiana benthamiana to Clavibacter michiganensis subsp. michiganensis
}

\author{
Vasudevan Balaji, Guido Sessa, and Christine D. Smart
}

First and third authors: Department of Plant Pathology and Plant-Microbe Biology, Cornell University, New York State Agricultural Experiment Station, Geneva 14456; and second author: Department of Plant Sciences, Tel-Aviv University, 69978 Tel-Aviv, Israel. Accepted for publication 27 October 2010.

\begin{abstract}
Balaji, V., Sessa, G., and Smart, C. D. 2011. Silencing of host basal defense response-related gene expression increases susceptibility of Nicotiana benthamiana to Clavibacter michiganensis subsp. michiganensis. Phytopathology 101:349-357.

Clavibacter michiganensis subsp. michiganensis is an actinomycete, causing bacterial wilt and canker disease of tomato (Solanum lycopersicum). We used virus-induced gene silencing (VIGS) to identify genes playing a role in host basal defense response to C. michiganensis subsp. michiganensis infection using Nicotiana benthamiana as a model plant. A preliminary VIGS screen comprising 160 genes from tomato known to be involved in defense-related signaling identified a set of 14 genes whose suppression led to altered host-pathogen interactions. Expression of each

of these genes and three additional targets was then suppressed in largerscale VIGS experiments and the effect of silencing on development of wilt disease symptoms and bacterial growth during an N. benthamiana-C. michiganensis subsp. michiganensis compatible interaction was determined. Disease susceptibility and in planta bacterial population size were enhanced by silencing genes encoding $N$. benthamiana homologs of ubiquitin activating enzyme, snakin-2, extensin-like protein, divinyl ether synthase, 3-hydroxy-3-methylglutaryl-coenzyme A reductase 2, and Ptolike kinase. The identification of genes having a role in the host basal defense-response to $C$. michiganensis subsp. michiganensis advances our understanding of the plant responses activated by $C$. michiganensis subsp. michiganensis and raises possibilities for devising novel and effective molecular strategies to control bacterial canker and wilt in tomato.
\end{abstract}

The gram-positive bacterium Clavibacter michiganensis subsp. michiganensis is the causal agent of bacterial canker and wilt disease in tomato (Solanum lycopersicum) (11). C. michiganensis subsp. michiganensis is a quarantine organism under international regulation, and can cause severe economic losses to tomato production worldwide. $C$. michiganensis subsp. michiganensis enters the plant from contaminated soil (where it can survive on plant residues up to 3 years) through root or stem wounds and natural openings, colonizes xylem vessels, and becomes systemic (26). $C$. michiganensis subsp. michiganensis colonization of xylem vessels leads to the appearance of typical disease symptoms that include unilateral wilting, leaflet necrosis, and lesions on stems. If infection occurs at the later stage of plant development, plants can survive and generate fruit that may develop bird's-eye spots, and seed could be contaminated with $C$. michiganensis subsp. michiganensis (18). At present, neither effective resistant tomato cultivars nor effective chemical controls are known. Therefore, disease prevention is mainly through use of pathogen-free certified seed and transplants, and rotation away from tomato for 3 years.

In recent years, important insight into the molecular mechanism of C. michiganensis subsp. michiganensis pathogenicity has been achieved (17), and the genome sequence of a C. michiganensis subsp. michiganensis strain has been completed (16). Major $C$. michiganensis subsp. michiganensis pathogenicity determinants, such as CelA encoding $\beta$-1,4-endocellulase (27) and

Corresponding author: C. D. Smart; E-mail address: cds14@cornell.edu

* The $\boldsymbol{e}$-Xtra logo stands for "electronic extra" and indicates that the online version contains two supplemental figures and two supplemental tables. Figure 1 appears in color online.

doi:10.1094/PHYTO-05-10-0132

(c) 2011 The American Phytopathological Society
Pat-1 encoding putative serine protease (13), are plasmid-borne; however, additional genes required for effective $C$. michiganensis subsp. michiganensis colonization of tomato plants are located in a chromosomal pathogenicity island of $\approx 125 \mathrm{~kb}(16)$. CelA and other extracellular enzymes produced by $C$. michiganensis subsp. michiganensis were hypothesized to cause degradation of cell walls in xylem vessels, resulting in impairment of water transport and consequent wilting $(17,27)$. The molecular function of Pat-1 and its possible plant protein targets have yet to be determined. A recent study demonstrated the interdependence of chromosomal and plasmid-located virulence gene expression in C. michiganensis subsp. michiganensis for a successful infection in tomato (6). Researchers also showed that expression of tomato basal defense-related genes, chitinase class II, and pathogenesisrelated (PR) protein-5 isoform was induced only in the absence of a large chp/tomA pathogenicity island (PAI) at early stages of $C$. michiganensis subsp. michiganensis infection, suggesting that PAI-located genes are involved in suppression of tomato basal defenses.

Host responses to $C$. michiganensis subsp. michiganensis infection and the molecular mechanisms associated with the development of disease symptoms caused by this pathogen are largely unknown. Using microarray analysis, we previously identified changes in the expression of 122 tomato genes at 8 days after inoculation with $C$. michiganensis subsp. michiganensis (2). During disease progress in this compatible interaction, expression of $\approx 93 \%$ of the differentially expressed genes was induced and identities of these genes suggested roles in basal defense for cell wall strengthening, protein degradation, oxidative burst, defenserelated hormones and signaling, transcription factors and many PR proteins (2). It was noteworthy that genes related to basal defense response were significantly enriched compared with their estimated frequency in the tomato genome (2), suggesting that the 
host elaborated an active basal defense reaction to counteract pathogen ingress. These genes include several receptor-like kinases and the putative extracellular glycoproteins ethylene-inducing xylanase (EIX1) (34) and tomato protein Ve1 that confers resistance to Verticillium wilt (15). In some cases, such as the recognition of bacterial flagellin by the receptor FLS2, pathogenassociated molecular patterns (PAMPs) triggered immunity limits for pathogen growth (43). Enhancing plant basal resistance may be an effective way to protect plants against diseases. It was shown that an early basal resistance inhibits tumor induction by Agrobacterium tumefaciens (40). Taken together, identification of genes that play a key role in eliciting basal defense response could help to devise strategies to reduce infection and protect host plants.

The development of high-throughput virus-induced gene silencing (VIGS) methods for Nicotiana benthamiana enables assessment of the role of a large number of candidate genes in hostpathogen interactions $(3,29)$. An advantage of VIGS, as previously noted, is the ability to assess function for genes whose mutation (or knockout) might be lethal in sexually propagated plants, or for genes that have functionally redundant family members (3). In this study, we evaluated whether the model host for plant pathology studies, $N$. benthamiana, was susceptible to $C$. michiganensis subsp. michiganensis. We subsequently used VIGS to investigate the role of basal defense response-related genes in the molecular compatible interaction between $C$. michiganensis subsp. michiganensis and $N$. benthamiana by assessing $C$. michiganensis subsp. michiganensis multiplication and wilt symptom appearance in the silenced plants. The possible functions of six genes identified by VIGS analyses in controlling C. michiganensis subsp. michiganensis-induced disease are discussed.

\section{MATERIALS AND METHODS}

Bacterial strains, plant material, and growth conditions. The bacterial strains GV2260 of A. tumefaciens and 04101 of $C$. michiganensis subsp. michiganensis (a New York field isolate) were used throughout this study. $N$. benthamiana seed were germinated in plastic pots $(10 \mathrm{~cm}$ in diameter $)$ containing potting mixture made of Cornell mix (a soilless peat mixture), vermiculite, and perlite in a ratio of 4:1:1. Two-week-old seedlings were transplanted to pots containing the above-mentioned mix, with one plant per pot. Plants were grown in a green house at $25 \pm 2^{\circ} \mathrm{C}$ and $70 \%$ relative humidity with a 16 - and 8 -h regime of light and darkness, respectively. Three-week-old plants at a four-leaf-stage were used in the silencing experiments.

$N$. benthamiana as a host for $C$. michiganensis subsp. michiganensis. In all, 45 7-week-old Tobacco rattle virus (TRV)only infected nonsilenced plants were inoculated by injecting a suspension of $C$. michiganensis subsp. michiganensis bacteria (1 $\times 10^{8} \mathrm{CFU} / \mathrm{ml}$ ) into the stem region at the third leaf from the bottom. The protocol for TRV inoculation is described below. Over a period of 20 days, 30 plants were monitored for development of canker and wilt symptoms while the remaining 15 plants were sacrificed for bacterial CFU estimation. In planta bacterial growth was measured five times $(4,8,12,16$, and 20 days postinoculation [dpi]) by harvesting stem pieces from three independent plants $(1 \mathrm{~cm}$ in length, one stem per plant). Tissue was ground in $10 \mathrm{mM} \mathrm{MgCl}$ and serially diluted samples of each stem extract were cultured on D2ANX medium plates containing nalidixic acid, polymixin sulfate, and cycloheximide at 28, 10, and $100 \mathrm{mg} / \mathrm{liter}$, respectively (9). Each stem piece was derived from an independent plant and was cut at $1 \mathrm{~cm}$ above the $C$. michiganensis subsp. michiganensis inoculation site. After incubation of the plates at $28^{\circ} \mathrm{C}$ for 3 to 4 days, the number of $\mathrm{CFU}$ per gram of tissue was determined for each sample. Mockinoculated plants were included as a negative control. The entire experiment was performed twice.
TRV vectors and plasmid constructs. TRV-based pTRV1 and pTRV2 VIGS vectors (29) were used in this study. In total, 160 tomato VIGS clones in a pTRV2 vector obtained from Drs. Sophia Ekengren and Gregory Martin (Boyce Thompson Institute, Ithaca, NY) were used for a preliminary VIGS screen in $N$. benthamiana to identify those genes that played a role in host basal defense response to $C$. michiganensis subsp. michiganensis and to determine the effect of silencing on $C$. michiganensis subsp. michiganensis multiplication and disease symptom development. Fourteen of these clones were selected for further study and sequenced in both directions using forward primer 5'-TTGTG TGTCAACAAAGATGG-3' $3^{\prime}$ and reverse primer 5'-GAACCTAAA ACTTCAGACACG-3' specific for pTRV2 plasmid. The insert sequences in the 14 clones were identified by blast analysis against the tomato expressed sequence tag (EST) databases in The Institute for Genomic Research (TIGR) and Solanaceae Genomics Network.

Three additional silencing targets-snakin-2, extensin-like protein $(E L P)$, and divinyl ether synthase $(D E S)$ - were amplified by reverse-transcription polymerase chain reaction (RT-PCR) on tomato first-strand complementary DNA (cDNA). Total RNA was extracted from stem tissues of tomato plants as described by the supplier's instructions (Qiagen Inc., Valencia, CA) and treated with RNase-free DNase. RNA ( $2.5 \mu \mathrm{g})$ was reverse transcribed for $50 \mathrm{~min}$ at $42^{\circ} \mathrm{C}$ in a $20-\mu \mathrm{l}$ reaction volume containing $100 \mathrm{U}$ of SuperScript II RNase H- reverse transcriptase (Invitrogen, Carlsbad, CA), $10 \mathrm{mM}$ dithiothreitol, $1 \times$ reverse transcriptase buffer (Invitrogen), $40 \mathrm{U}$ of RNase inhibitor (Invitrogen), $500 \mu \mathrm{M}$ each $\mathrm{dNTP}$, and $500 \mathrm{ng}$ of oligo (dT) 18-24 primer. The reaction was stopped by incubation at $70^{\circ} \mathrm{C}$ for $15 \mathrm{~min}$. RT reaction mixture $(1 \mu \mathrm{l})$ was used for PCR in a $25-\mu$ l reaction volume containing 1 unit of Taq DNA polymerase (New England Biolabs, Ipswich, MA), $200 \mu \mathrm{M}$ each dNTP, and $300 \mathrm{nM}$ forward and reverse primers for each gene. A 309-bp fragment for snakin-2 was amplified using forward primer 5'-CGCGGATCCTGGAACTT GTTGTGCTAGA-3' and reverse primer 5'-GCTCTAGACCAG AACAAATATCACACC-3'. A 383-bp fragment of tomato ELP (2) was amplified using forward primer 5'-GCTCTAGATGTA GTCTCCATCAAGTGGTCA-3' and reverse primer 5'-CGC GGATCCGCAGCTTAATTTTACTCCTTGG-3'. A 554-bp fragment of DES was amplified using forward primer 5'-CGC GGATCCGTGAAATTCCAGGGGACTATG- $3^{\prime}$ and reverse primer 5'-GCTCTAGAAGCGCCAAGAACT GTATCAGA-3'. The cDNA fragments were double digested with BamHI/XbaI and cloned into the corresponding sites of pTRV2. The identity of these constructs was confirmed by restriction analysis and nucleotide sequencing. The pTRV2 derivatives were introduced into $A$. tumefaciens strain GV2260 by electroporation and the agrotransformants were confirmed by restriction analysis of the introduced plasmid.

Virus infection by Agrobacterium spp.-mediated infiltration. Agroinoculations for VIGS were performed using the leaf infiltration method as previously described (29). GV2260 containing pTRV1 or pTRV2 and its derivatives were mixed in a 1:1 ratio and inoculated into the lower leaves of 3-week-old $N$. benthamiana plants by leaf infiltration using a needle-less syringe. For the preliminary screen with 160 clones, 4-week-old $N$. benthamiana plants were used. Plants were left in the greenhouse for growth and viral spread.

High through-put preliminary screens. For the preliminary screen with 160 clones, four plants per clone were used, and inoculated with TRV as described above. These plants were inoculated by infiltrating two leaves per plant with a $C$. michiganensis subsp. michiganensis suspension $\left(1 \times 10^{8} \mathrm{CFU} / \mathrm{ml}\right)$. The infiltration site was monitored daily for 10 days following inoculation.

Seventeen clones were included in the next experiment to further examine the developmental phenotype due to silencing of these potential basal defense-related genes (Table 1). In this experiment, 14 3-week-old plants were individually inoculated 
with pTRV1 and the appropriate pTRV2-EST fragment as described above. Treatments were assigned in a completely random design. The constructs pTRV2:SIPDS (phytoene desaturase from $S$. lycopersicum) and pTRV2 were introduced as positive and negative controls, respectively. Plants were observed daily for 2 weeks to identify silencing-associated phenotypes.

Determination of host susceptibility in silenced plants. Eleven genes (Table 1, genes not in bold) were included in these VIGS studies to determine the possible role of each gene in basal defense to $C$. michiganensis subsp. michiganensis by silencing 12 3 -week-old plants for each clone. Treatments were assigned in a completely random design. Two weeks after virus infection, silenced plants were inoculated with a suspension of C. michiganensis subsp. michiganensis at $1 \times 10^{8} \mathrm{CFU} / \mathrm{ml}$ in the stem region at the third leaf from the bottom and monitored over a period of 20 days for the appearance and progress of wilt symptoms. Mock-inoculated control plants were included for each clone, and empty-vector C. michiganensis subsp. michiganensisinoculated plants were used as positive controls. The entire experiment was performed twice. To get a quantitative estimation of the silencing effect on $C$. michiganensis subsp. michiganensis multiplication and of the timing of wilt symptom onset, we applied a parameter known as wilting index (WI), which has been previously defined as the number of days after infection at which $50 \%$ of the plants display the first wilt symptoms $(2,31)$.

Host susceptibility and bacterial multiplication in plants silenced for six genes involved in basal defense. Two independent biological repeats were performed for six genes found to be involved in basal defense against $C$. michiganensis subsp. michiganensis. In each repeat, for each of the six target genes plus the vector-only control, 60 plants were silenced and inoculated with $C$. michiganensis subsp. michiganensis as described above. Treatments were assigned in a completely random design. Thirty of the plants were monitored daily for 20 days for the development of wilt symptoms and used to calculate the WI as described above. The other 30 plants were used to determine the in planta $C$. michiganensis subsp. michiganensis population size and to extract RNA for transcript analysis (described below). The entire experiment was repeated twice. WI data for each replicate were combined and subjected to analysis of variance (ANOVA) using the Mixed Models (MIXED) procedure of SAS (version
9.2; SAS Institute Inc., Cary, NC). Treatment effects were considered statistically significant at $\alpha=0.05$.

In planta bacterial growth was measured at two time points (4 and $8 \mathrm{dpi}$ ) by grinding three stem pieces $(1 \mathrm{~cm}$ in length, one stem per plant) in $10 \mathrm{mM} \mathrm{MgCl}_{2}$ and plating serially diluted samples of each stem extract on D2ANX medium (9). Each stem piece was derived from an independent silenced plant and was cut at $1 \mathrm{~cm}$ above the $C$. michiganensis subsp. michiganensis inoculation site. After incubation of the plates at $28^{\circ} \mathrm{C}$ for 3 to 4 days, the number of CFU per gram of tissue was determined for each sample. Data were analyzed using Student's $t$ test, and treatment effects were considered statistically significant at $\alpha=0.05$.

Semiquantitative RT-PCR. Total RNA was isolated from TRV-only infected plants and those silenced for the six target genes with the RNeasy Plant Mini Kit (Qiagen Inc.) according to the manufacturer's instructions and treated with RNase-free DNase. For each treatment, a pool of eight 1-cm-long stem pieces, cut from eight independent plants (one stem per plant) at the third leaf from the bottom were used for RNA extraction. Stem tissues were harvested from plants 2 weeks post-VIGS. Total RNA $(2.5 \mu \mathrm{g})$ was reverse transcribed as previously described. PCR was performed in 100- $\mu$ l reactions using $3 \mu \mathrm{l}$ of reverse transcription mix containing the first-strand cDNA, 1× PCR buffer containing $1.5 \mathrm{mM} \mathrm{MgCl} 2,200 \mu \mathrm{M}$ each dNTP, 300 $\mathrm{nM}$ of each gene-specific primer (Supplementary Table S2), and $2 \mathrm{U}$ of Taq DNA polymerase (New England Biolabs, Ipswich, MA). To ensure that equal amounts of cDNA were used for silenced and nonsilenced plants, parallel reactions were run with $N$. benthamiana elongation factor $\alpha$ (a house keeping gene) (NbEF- $\alpha$; AY206004) primer as control. Each PCR reaction included an initial denaturation at $94^{\circ} \mathrm{C}$ for $2 \mathrm{~min}$, followed by cycling conditions of $94^{\circ} \mathrm{C}$ for $30 \mathrm{~s}$, annealing for $30 \mathrm{~s}$ (temperature specified in Supplementary Table S2 for each gene), and an extension at $72^{\circ} \mathrm{C}$ for $1 \mathrm{~min}$. A $10-\mu \mathrm{l}$ aliquot was removed from each reaction after $20,25,30,35,40,45$, and 50 cycles, respectively. The aliquots were analyzed on a $1.2 \%$ agarose gel stained with ethidium bromide. The intensity of PCR products corresponding to target genes was quantified at 30 and 50 cycles in TRV-only infected control and silenced plants using a Quantity One Version 4.6.9 software (Bio-Rad Laboratories, Hercules, CA). The entire experiment was repeated.

TABLE 1. Supporting information for the genes targeted by virus-induced gene silencing in Nicotiana benthamiana ${ }^{\mathrm{a}}$

\begin{tabular}{|c|c|c|c|c|}
\hline Gene & Fragment length $(b p)^{b}$ & TC no. ${ }^{\mathrm{c}}$ & Nucleotide identity $(\%)^{\mathrm{d}}$ & Length $(\mathrm{nt})^{\mathrm{e}}$ \\
\hline Elongation factor & 689 & TC14415 & 79 & 41 \\
\hline Heat shock protein $90^{\mathrm{f}}$ & 1,691 & NP880339 & 85 & 73 \\
\hline Eukaryotic initiation factor & 996 & TC13235 & 80 & 50 \\
\hline$\beta$-Tubulin & 752 & No hits found & $\ldots$ & $\ldots$ \\
\hline $60 S$ ribosomal protein & 813 & TC12372 & 91 & 41 \\
\hline 40S ribosomal protein & 737 & TC16301 & 78 & 26 \\
\hline Coumarate-CoA ligase & 1,892 & CK299019 & 86 & 31 \\
\hline Uridine diphospho-xylose synthase & 2,109 & NP864107 & 89 & 44 \\
\hline Unknown protein & 1,175 & No hits found & $\ldots$ & $\ldots$ \\
\hline$D N A J$ & 525 & CK285070 & 89 & 61 \\
\hline Ribulose-1 5-bisphosphate carboxylase small subunit precursor ${ }^{\mathrm{f}}$ & 463 & TC15331 & 96 & 154 \\
\hline Pto-like serine/threonine kinase & 2,368 & TC16220 & 82 & 27 \\
\hline Ubiquitin activating enzyme & 447 & EH367417 & 86 & 39 \\
\hline 3-Hydroxy-3-methylglutaryl-coenzyme A reductase $2^{\mathrm{f}}$ & 420 & TC11881 & 99 & 220 \\
\hline Snakin-2 & 309 & TC14694 & 82 & 24 \\
\hline Extensin-like protein & 383 & TC13329 & 75 & 27 \\
\hline Divinyl ether synthase & 554 & CK293631 & 84 & 27 \\
\hline
\end{tabular}

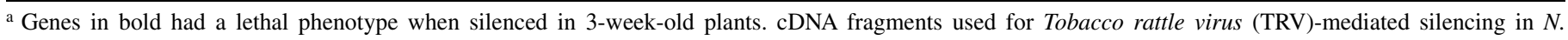
benthamiana were isolated from tomato.

${ }^{\mathrm{b}}$ Length of the cDNA fragments cloned in the TRV2 vector used for silencing.

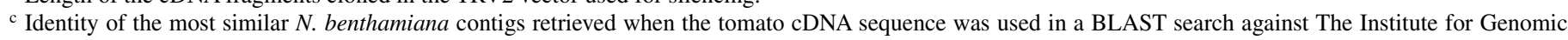
Research $N$. benthamiana gene index (http://compbio.dfci. harvard.edu/tgi/cgi-bin/tgi/Blast/index.cgi).

${ }^{\mathrm{d}}$ Overall sequence identity (percentage) between the tomato and $N$. benthamiana genes.

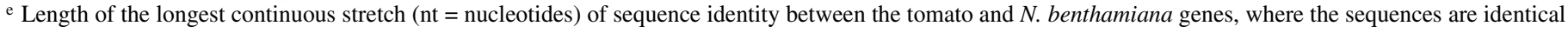
between the two genes.

${ }^{\mathrm{f}}$ Genes with additional homologs that could be affected by co-silencing. 


\section{RESULTS}

N. benthamiana as a model host for $C$. michiganensis subsp. michiganensis. Though a VIGS system has been established for tomato (14), it is not as robust as VIGS in N. benthamiana, which

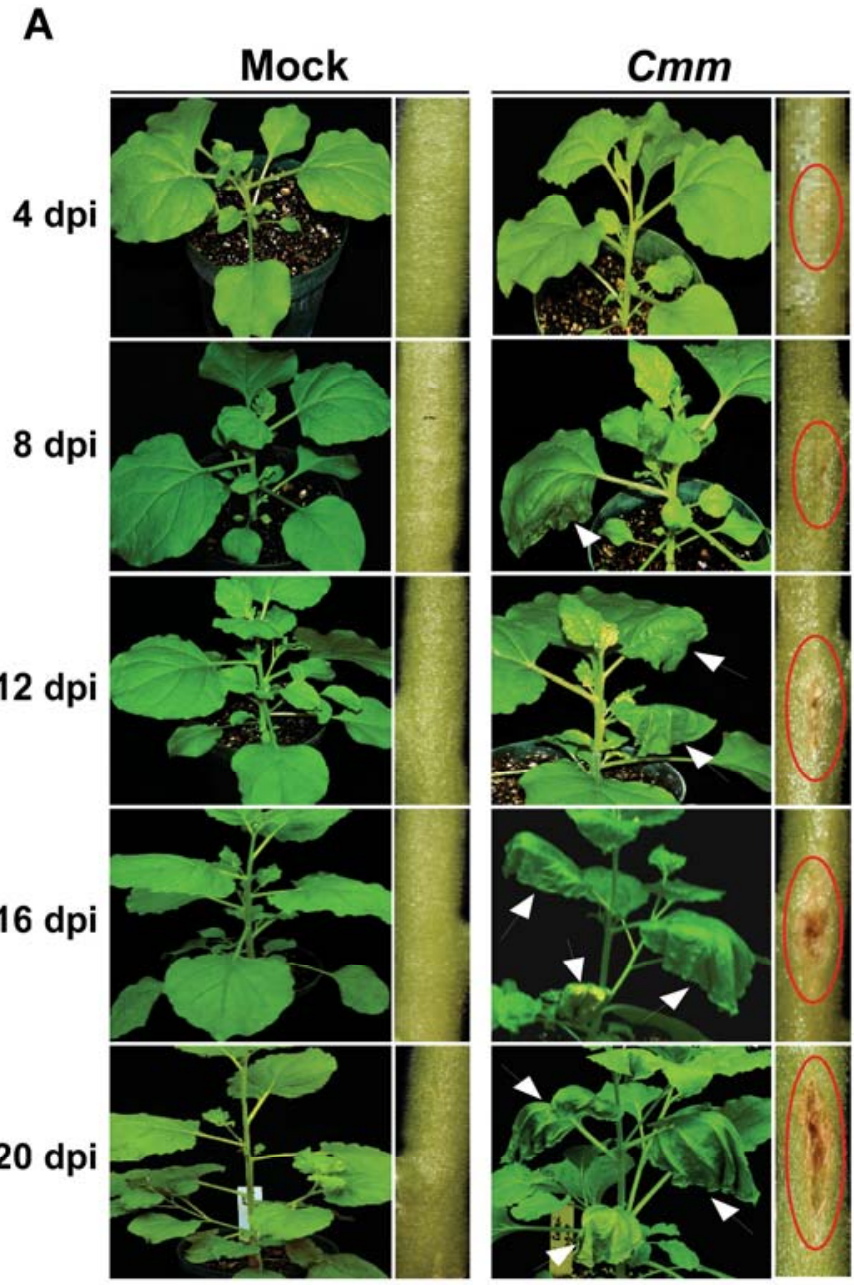

B

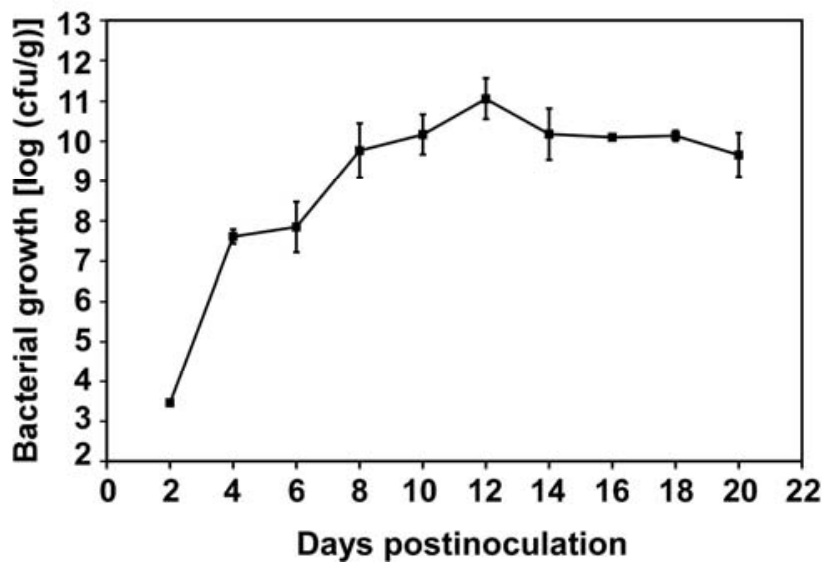

Fig. 1. Disease symptoms and bacterial growth in Nicotiana benthamiana plants inoculated with Clavibacter michiganensis subsp. michiganensis. Fiveweek-old plants were inoculated with a suspension of $C$. michiganensis subsp. michiganensis bacteria at a titer of $10^{8} \mathrm{CFU} / \mathrm{ml}$ in the stem at the third leaf from the bottom stem or mock inoculated. A, Leaves with wilt symptoms (indicated by arrows) and the plant stem showing canker symptoms (encircled areas) were photographed at $4,8,12,16$, and 20 days postinoculation (dpi). B, Bacterial population size determined from stem samples harvested at different time points during a period of $20 \mathrm{dpi}$. Data represent the mean \pm standard deviation $(n=3)$. is highly efficient, uniform, and used routinely for large-scale screening of candidate genes in host-pathogen interactions. There are no previous studies where $N$. benthamiana has been used a model host for $C$. michiganensis subsp. michiganensis. Thus, the high susceptibility of $N$. benthamiana to VIGS combined with the fact that tomato ESTs can effectively knock down gene expression in $N$. benthamiana (12) prompted us to deploy it as a model host for $C$. michiganensis subsp. michiganensis. To this end, an experimental system was developed to achieve coordinated and reproducible infection of $N$. benthamiana plants by $C$. michiganensis subsp. michiganensis. At 4 dpi, canker lesions (Fig. 1A) were visible at the inoculation site in the stem of $50 \%$ of a set of 30 C. michiganensis subsp. michiganensis-inoculated plants, whereas wilt symptoms were not yet apparent. At 8 dpi, 30\% of the $C$. michiganensis subsp. michiganensis-inoculated plants showed initial wilt symptoms (Fig. 1A), and the canker lesions started to enlarge by spreading in both directions from the inoculation site and appeared on the stems of all C. michiganensis subsp. michiganensis-inoculated plants. At $12 \mathrm{dpi}, 45 \%$ of the inoculated plants showed typical wilt symptoms. At 16 dpi, 60\% of the inoculated plants exhibited severe wilt symptoms. By $20 \mathrm{dpi}, 70 \%$ of the inoculated plants exhibited clear and advanced wilt symptoms. A mock-inoculation treatment was also included as a control and the mock-inoculated plants remained symptomfree throughout the course of the experiment (Fig. 1A). To establish a correlation between the appearance of disease symptoms and bacterial CFU, C. michiganensis subsp. michiganensis growth in stem tissues above the inoculation site was monitored over the 20-day period. Bacterial multiplication was exponential during the first 10 days postinfection and approached saturation at 12 dpi (Fig. 1B), with the stem tissue colonized with C. michiganensis subsp. michiganensis at a titer of $10^{10}$ to $10^{11} \mathrm{CFU} / \mathrm{g}$. The experiment was repeated with consistent results (data not shown). Taken together, these results showed that, in our experimental system, initial canker lesions appeared when C. michiganensis subsp. michiganensis was in the exponential phase and still multiplying in infected stems, whereas advanced leaf wilting and formation of enlarged cankers was observed when the bacterial titer approached saturation.

VIGS in $N$. benthamiana using tomato orthologs involved in plant defense responses affects $C$. michiganensis subsp. michiganensis infection. To identify host genes affecting C. michiganensis subsp. michiganensis multiplication and appearance of disease symptoms, the TRV-based VIGS vector system was used to silence candidate genes in 4-week-old $N$. benthamiana. Nearly 160 genes identified to be involved in a resistance response of tomato to Pseudomonas syringae pv. tomato (speck disease pathogen) (32) were tested in a preliminary screen. Of these, 14 resulted in an altered response wherein the silenced plants developed rapid necrotic lesions in the $C$. michiganensis subsp. michiganensis infiltrated zone. In the modified interaction, silenced plants responded to the $C$. michiganensis subsp. michiganensis inoculation $\approx 24 \mathrm{~h}$ earlier than control TRV-only nonsilenced plants (data not shown). For further VIGS analysis, three additional targets (ELP, snakin-2, and DES) were selected.

Because the TRV clones were not derived from $N$. benthamiana and effective silencing by VIGS requires a 21- to 23-bp minimal region of nucleotide identity (39), we performed BLAST analyses with the sequences of each of the 17 tomato cDNA fragments against the TIGR database of $N$. benthamiana ESTs. For 14 of the 17 genes, a single $N$. benthamiana ortholog with a highly significant sequence similarity was identified (Table 1). One shortcoming of VIGS and other silencing technologies is the possibility that closely related nontarget genes are co-silenced $(4,25)$. Three genes in this study had multiple $N$. benthamiana homologs with a 21-bp or longer region identical to the tomato gene sequence (Table 2). Therefore, it is possible that these homologs could be silenced as well. 
Developmental phenotypes revealed by silencing tomato ESTs in $N$. benthamiana. For each of the 17 clones identified in the preliminary screen, VIGS was performed on 143 -week-old $N$. benthamiana plants, and plant development was monitored for 4 weeks. The constructs pTRV2:SIPDS and pTRV2 were introduced as positive and negative control, respectively. Silencing of the endogenous $P D S$ gene, which causes photobleaching, was used as a control for VIGS efficiency. In two independent experiments, even at 2 weeks post-agroinfiltration, the leaves and stem exhibited a complete photobleach phenotype in $N$. benthamiana plants silenced for the PDS gene (data not shown). Based on these results, 2-week-old silenced plants were used for all C. michiganensis subsp. michiganensis bioassays. For 12 of the 17 clones, silencing of the $N$. benthamiana genes resulted in a developmental phenotype, the most frequent of which was severe stunting followed by death of the silenced plants (Supplementary Figure S1A; Supplementary Table S1). The silencing phenotypes were apparent at 2 weeks post-agroinfiltration. The stunting and death phenotype associated with silencing of these six genes (Supplementary Figure S1A) was also observed in the preliminary VIGS screen but it occurred much later because, in those preliminary experiments, the treated plants were older (4week-old plants were used for VIGS), which allowed leaf infiltration with $C$. michiganensis subsp. michiganensis before the plants started to decay. The six clones that induced this lethal phenotype were excluded from further analyses.

Silencing of some genes led to developmental alterations that might result from a defective meristem functioning. Reduction in levels of the coumarate-CoA ligase, uridine diphospho-xylose synthase $(U D P-X S)$, unknown protein $(U N P)$, and $D N A J$ resulted in an attenuation of plant growth and severe stunting (Supplementary Figure S1B). Another phenotype observed was the mild deformation (crinkling) of the upper, presumably silenced, leaves, as shown for 3-hydroxy-3-methylglutaryl-coenzyme A reductase $2(H M G R)$ and ubiquitin activating enzyme (UAE) (Supplementary Figure S1B). Suppression of Pto-like kinase $(P L K)$, snakin-2, ELP, ribulose-1,5-bisphosphate carboxylase small subunit precursor $(R B C S)$, and $D E S$ did not lead to any visible morphological changes (Supplementary Figure S1C).

Determination of host susceptibility in VIGS plants. We next evaluated the possible role of each of the remaining 11 genes in basal defense responses to $C$. michiganensis subsp. michiganensis infection. Silenced plants were inoculated with $C$. michiganensis subsp. michiganensis and monitored over a period of 20 days for the appearance and progress of wilt symptoms. Mock-inoculated control plants remained symptomless throughout the course of the experiment, and $C$. michiganensis subsp. michiganensis-inoculated TRV empty-vector control plants were used as positive controls. The WI was determined for each of the 11 silenced genes and the controls. Whereas the WI for control C. michiganensis subsp. michiganensis-inoculated plants was 19 days, plants silenced for $N$. benthamiana homologs of snakin-2, ELP, $D E S, U A E, H M G R$, and PLK had a WI of 6 to 14 days, demonstrating a significantly earlier onset of wilting. Plants silenced for the other five genes (coumarate-CoA ligase, UDP-XS, UNP, DNA $J$, and $R B C S$ ) developed wilt symptoms much later than the $C$. michiganensis subsp. michiganensis-inoculated control plants. These five VIGS clones were initially identified from the preliminary VIGS screen, where they responded $24 \mathrm{~h}$ earlier than control plants to $C$. michiganensis subsp. michiganensis infiltration in the leaves, exhibiting an early necrotic response. However, in this large-scale analysis, a quantitative scoring method was applied by using the WI to determine the effect of silencing on the development of characteristic wilt symptoms. The quantitative analysis revealed that silencing of these five genes delayed wilting when compared with the nonsilenced

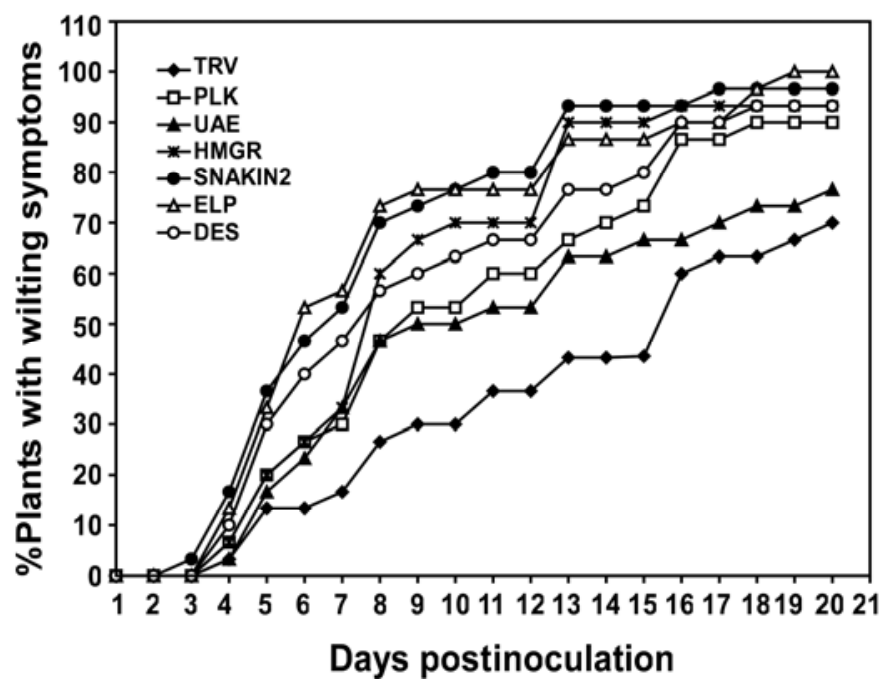

Fig. 2. Effect of silencing on development of wilt symptoms in Nicotiana benthamiana plants infected with Clavibacter michiganensis subsp. michiganensis. Two weeks post-silencing, plants were inoculated with a C. michiganensis subsp. michiganensis suspension $\left(10^{8} \mathrm{CFU} / \mathrm{ml}\right)$ and examined for development of wilt symptoms during a 20-day period. Percentage of plants showing wilt symptoms was calculated in a group of 30 plants for each of the silenced genes and the Tobacco rattle virus (TRV)-only infected control. Data shown are representative of two independent experiments for each silenced gene. Wilting index (WI), defined as the number of days after inoculation at which $50 \%$ of the total plants show first wilting symptoms, were as follows: TRV, 16; Pto-like kinase $(P L K), 9$; ubiquitin activating enzyme $(U A E), 9$; 3-hydroxy-3-methylglutaryl-coenzyme A reductase 2 (HMGR ), 8; snakin-2, 7; extensin-like protein $(E L P), 6$; and divinyl ether synthase $(D E S), 8$.

TABLE 2. Comparison of the most closely related paralogs of three silenced genes

\begin{tabular}{|c|c|c|c|c|}
\hline Nicotiana benthamiana orthologa & N. benthamiana paralogs or off-targets ${ }^{b}$ & TC no. ${ }^{c}$ & Nucleotide identity $(\%)^{\mathrm{d}}$ & Length $(\mathrm{nt})^{\mathrm{e}}$ \\
\hline HSP9O & $H S P 90 b$ & TC16336 & 82 & 71 \\
\hline \multirow[t]{2}{*}{$R B C S$} & $R B C S b$ & CN748449 & 87 & 59 \\
\hline & $R B C S c$ & CN748824 & 82 & 40 \\
\hline \multirow[t]{3}{*}{$H M G R$} & $C A^{\mathrm{f}}$ & NP375379 & 94 & 39 \\
\hline & $40 S-R P^{\mathrm{f}}$ & EC277789 & 90 & 29 \\
\hline & $E I F-4 A^{\mathrm{f}}$ & EC277784 & 72 & 39 \\
\hline
\end{tabular}

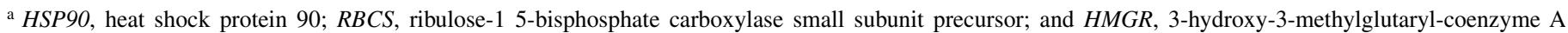
reductase 2 . See Table 1 for contig numbers for $N$. benthamiana ortholog.

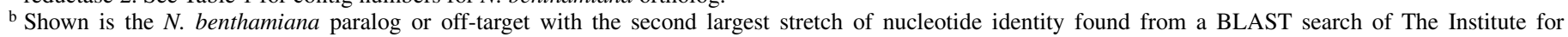
Genomic Research $N$. benthamiana gene index.

c Gene identity of the closest paralog or off-target.

d Sequence identity (in percentage) between the tomato and $N$. benthamiana paralog or off-target.

e Longest continuous stretch ( $\mathrm{nt}=$ nucleotides) where the sequences are identical between the tomato and $N$. benthamiana paralog or off-target.

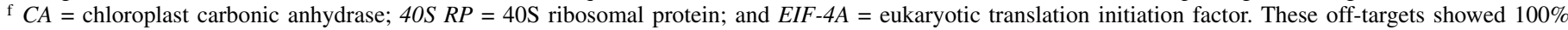
identity in a single continuous nucleotide stretch of $>21 \mathrm{bp}$. Percent sequence identity scored based on the matching nucleotide numbers are $48 / 51$ nucleotides (nt) $(94 \%), 50 / 55 \mathrm{nt}(90 \%)$, and 84/116 nt (72\%) for CA, 4OS RP, and EIF-4A, respectively. 
control plants. These silenced plants did not have a WI during a disease monitoring period of 20 days when control plants had a WI of 19 days. We observed a similar pattern for these five genes in an independent experiment where the silenced plants did not show a WI and the control nonsilenced TRV-only infected plants showed a WI of 17 days. Because these genes were not involved in host basal defense, they were not carried on in this study.

Silencing of six genes enhances $C$. michiganensis subsp. michiganensis susceptibility in $\boldsymbol{N}$. benthamiana. With the six remaining genes, two independent large-scale biological repeats were carried out. In each repeat, for each of the six target genes and the TRV vector-only control, 60 plants were silenced, half of which were allotted to monitor the development of wilt symptoms and to calculate the WI. The other half was used to determine the in planta $C$. michiganensis subsp. michiganensis population size and to extract RNA for transcript analysis. In these experiments, the inoculated control plants showed a WI of 16 days (Fig. 2). In both repeats, plants that had been silenced for one of the six genes showed wilt symptoms sooner than the control plants, with a WI of 7 to 12 days, depending on the silenced gene. The WI of all VIGS treatments were significantly different from the control TRV-only plants at $\alpha=0.05$.

Increased disease susceptibility is correlated with VIGSmediated suppression of target gene expression. Two weeks after TRV inoculation, for each of the six genes, total RNA was isolated from a pool of stem tissues harvested from eight independently silenced plants and the relative abundance of the target gene transcripts was determined by semiquantitative RT-PCR

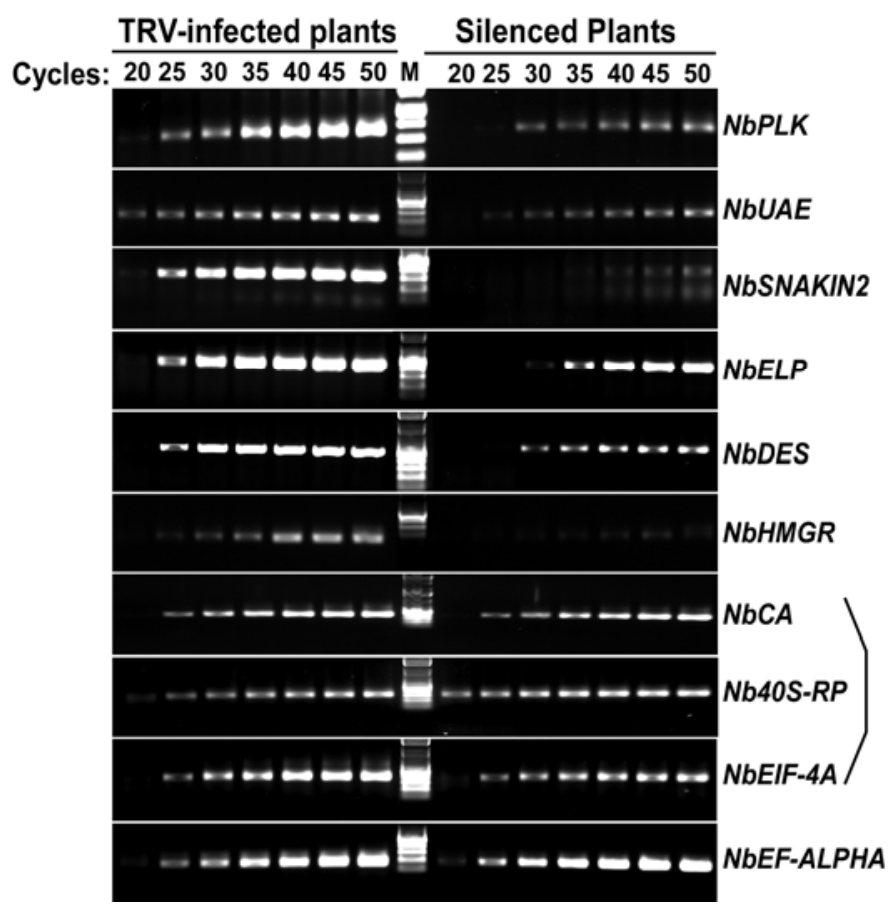

Fig. 3. Semiquantitative reverse-transcription polymerase chain reaction (RTPCR) to demonstrate virus-induced gene silencing-mediated suppression of specific RNA transcripts in Nicotiana benthamiana. Total RNA was isolated from Tobacco rattle virus (TRV)-only infected plants or plants silenced for specific genes (listed on right) and was used to generate first-strand complementary DNA (cDNA). cDNA was used in an RT-PCR using primers specific to the target gene. Off-targets for gene $N b H M G R$ with one $\geq 22$-bp stretch of $100 \%$ identity is shown in brackets. To compare the abundance of transcripts between silenced and nonsilenced plants, PCR products were sampled from each PCR cycle number indicated. RT-PCR products were separated on a $1 \%$ agarose gel and visualized after staining with ethidium bromide. Bottom panel in the figure shows the amount of elongation factor $\alpha(\mathrm{NbEF}-\alpha)$ transcripts in TRV-only infected plants as well as plants silenced for divinyl ether synthase $(N b D E S)$. This control was performed for every gene-silenced plant with similar results (data not shown). using primers designed against the $N$. benthamiana orthologs (Supplementary Table S2). A clear reduction was observed in the transcript levels of each of the genes targeted by VIGS in the silenced plants when compared with the control plants (Fig. 3). To evaluate the silencing efficiency, we quantified the intensity of the ethidium bromide-stained band corresponding to the target gene in silenced and TRV-only infected control plants after 30 cycles in the RT-PCR reaction. VIGS efficiency, represented as percent reduction, was 70 to $99 \%$ depending on the gene silenced (PLK, 70\%; UAE, 81\%; DES, 84\%; ELP, 92\%; HMGR, 96\%; and snakin-2, 99\%). Reduction in the transcript level in silenced plants was also evident upon quantification of band intensities after 50 cycles (data not shown). These data were confirmed in an independent biological repeat (data not shown). These experiments demonstrate that the tomato sequences were effective in silencing the $N$. benthamiana orthologs.

In the case of $H M G R$ silencing, we found three potential offtargets that had a single region of $\geq 21$ bp with perfect sequence identity (Table 2). Because this sequence identity might allow these off-target genes to be silenced by the tomato cDNA fragment, we developed gene-specific primers for each of them and examined their transcript abundance in $H M G R$-silenced plants and control plants. In each case, in spite of the nucleotide identity over a $>21$-bp region, we found no decrease in transcript abundance for these off-targets (Fig. 3), which was further confirmed by amplicon intensity quantification analysis. Thus, although we cannot exclude the silencing of very closely related paralogous genes in all cases, these data support a high degree of silencing specificity for the $N$. benthamiana orthologs of our tomato gene set.

Enhanced disease susceptibility is associated with increased C. michiganensis subsp. michiganensis titers. To gain insight into the basis for increased disease susceptibility, we analyzed $C$. michiganensis subsp. michiganensis titers in individual stems of infected plants silenced for each of the six genes and of control plants at two time points: at $4 \mathrm{dpi}$, when bacterial growth in inoculated stems of control plants is logarithmic and canker lesions are the only visible disease symptom; and at $8 \mathrm{dpi}$, when distinctive wilt and canker symptoms are visible on leaves and stems, respectively (Fig. 1). Although, at $4 \mathrm{dpi}$, no significant differences could be noted, at $8 \mathrm{dpi}$, in all cases, the increase in the bacterial populations was much larger and significant in the silenced plants compared with that in the control plants (Fig. 4). In the control plants, the increase in the $C$. michiganensis subsp. michiganensis population between 4 and 8 dpi was 70 -fold. In the silenced plants, the largest increases in CFU were noted for $U A E$ and snakin-2 (4,000- and 3,000-fold), followed by HMGR and $D E S$ (1,500- and 800-fold), ELP (500-fold) and, finally, the smallest increase was in $P L K$-silenced plants (130-fold) (Fig. 4). A similar pattern of increase in bacterial titer in silenced plants versus control was observed in an independent biological repeat, with the smallest increase in CFU observed for PLK-silenced plants when compared with other target genes (Supplementary Figure S2). Based on these results, it seems that the WI (Fig. 2) and bacterial titer (Fig. 4) are not strictly inversely proportional. However, it should be noted that the WI was calculated from a set of 30 plants and bacterial titer was determined from a group of three representative plants for each silencing treatment. It is likely that a variation in the bacterial titer between the independent silenced plants in a group of 30 plants contributes to the final WI. Overall, silenced plants had significantly higher bacterial populations and developed wilt symptoms much earlier than control, nonsilenced plants.

\section{DISCUSSION}

$N$. benthamiana is susceptible to a wide range of plantpathogenic agents such as viruses, bacteria, and fungi, making 
this species a widely used model for host-pathogen research, particularly in the context of innate immunity and defense signaling. The development of plant virus-based vectors quickly led to the discovery of VIGS technology (39) that transformed $N$. benthamiana into a powerful reverse-genetics system $(12,29)$. Although a VIGS system has been established for tomato, the efficiency of silencing and level of desired transcript suppression is still low and less uniform compared with $N$. benthamiana (35). For this study, $N$. benthamiana was selected because it is a wellestablished host for high-throughput VIGS and enables assessment of the role of a large number of candidate genes in hostpathogen interactions. Importantly, $N$. benthamiana ESTs are generally highly homologous to those of agriculturally relevant solanaceous crops, such as tomato, potato, and pepper. Thus, functional genomics projects concerned with host-pathogen interactions conducted in $N$. benthamiana are believed to yield a framework of genes that play similar roles in agronomically important crops. However, we cannot rule out the fact that, though $N$. benthamiana ESTs are generally highly homologous to tomato, there is likelihood for variation or a totally different disease phenotype in silenced tomato (natural host of $C$. michiganensis subsp. michiganensis) when compared with our results on $N$. benthamiana. This possibility will be verified in future studies.

Although $N$. benthamiana is a host of many plant-pathogenic agents, it had not been shown to be susceptible to $C$. michiganensis subsp. michiganensis. This study demonstrates for the first time the induction of wilt and canker symptoms in $N$. benthamiana characteristic of $C$. michiganensis subsp. michiganensis infection in its natural host, tomato. We took advantage of the accessibility of $N$. benthamiana to the VIGS methodology to screen tomato genes known to be involved in the host defense response for their putative role during the interaction with $C$. michiganensis subsp. michiganensis. It was particularly interesting that the silencing of six genes increased susceptibility to $C$. michiganensis subsp. michiganensis. Although the level of susceptibility conferred varied among the genes tested, as shown by the difference in the bacterial titer and WI, it is possible that different degrees of silencing for each of the genes contributed to the different levels of observed susceptibility.

Our data indicate that $N$. benthamiana homologs of tomato ELP, snakin-2, UAE, DES HMGR, and PLK play a role in host basal defense response to $C$. michiganensis subsp. michiganensis. Research on a number of host-pathogen systems has shown that extensin levels increase in response to pathogen ingress and proliferation $(10,24)$. A recent study convincingly demonstrated a functional linkage between extensin synthesis and plant resistance to pathogen attack (41). They showed that EXT1 extensin-overexpressing transgenic Arabidopsis limits pathogen invasiveness and were resistant to $P$. syringae pv. tomato DC3000. More recently, our work on transcriptome analysis of the tomato- $C$. michiganensis subsp. michiganensis compatible interaction revealed that the tomato ELP encoding cDNA was upregulated 12to 20 -fold at 4 and $8 \mathrm{dpi}$ after $C$. michiganensis subsp. michiganensis infection (2). The data reported here clearly indicate that silencing an $N$. benthamiana ELP enhanced susceptibility to $C$. michiganensis subsp. michiganensis infection and induced early wilting.

Our findings also demonstrate that the silencing of snakin-2 enhanced susceptibility to $C$. michiganensis subsp. michiganensis and advanced wilt symptom development. Furthermore, our data confirm the previous observations on the antimicrobial role of snakin-1 and snakin-2 peptides in defense response to bacterial and fungal pathogens $(5,28,37)$. It is interesting that purified snakin-2 peptide from potato tubers caused rapid aggregation and inhibited growth of $C$. michiganensis subsp. sepedonicus, a species closely related to $C$. michiganensis subsp. michiganensis and the causative agent of potato ring rot disease (5). Recently, it was shown that overexpression of the snakin-1 gene enhanced resistance in transgenic potato plants to Erwinia carotovora and Rhizoctonia solani (1). Considering its role as an antimicrobial peptide and based on our results reported here, it is tempting to speculate that snakin-2 could serve as a promising candidate to reduce $C$. michiganensis subsp. michiganensis infection and disease development.

Increased susceptibility to $C$. michiganensis subsp. michiganensis was also evident in plants silenced for $U A E$ and DES. $U A E$ is the first in the multi-enzymatic system that operates ubiquitination, which has been shown to be important in plant defense (30,33). A recent study (22) identified a modifier of sncl 5 (mos5) mutant in Arabidopsis, which carries a mutation in ubiquitin-activating enzyme 1 that suppresses suppressor of nprl-1 constitutive 1 (sncl)-mediated constitutive resistance and exhibits enhanced susceptibility to virulent pathogen and is impaired in response to infection with avirulent bacteria. The mos5 gene encodes an essential component of the ubiquitin pathway and implicates a requirement for ubiquitination in resistance-protein signaling (22). It is interesting to note that $U A E$ was upregulated by 70 -fold in our microarray analysis of the tomato- $C$. michiganensis subsp. michiganensis compatible interaction (2). The mos 5 results (22) along with the $U A E$ results from this study suggest a conserved role for $U A E$ in basal defense. It has been shown that divinyl ethers colneleic acid and colnelenic acid derived by the sequential action of a 9-LOX (lipoxygenase) and a 9-DES accumulate in tobacco or potato in response to elicitor treatment or pathogen ingress $(19,21,36)$. 9-LOX-silenced potato plants show decreased levels of 9-LOX-derived oxylipins in leaves inoculated with the incompatible pathogen $P$. syringae pv. maculicola (20). Genes encoding allene oxide synthase and LOX were induced four- to sevenfold in our previous microarray analysis (2), suggesting a role for oxylipins in the basal defense reaction to $C$. michiganensis subsp. michiganensis attack. Those data, combined with the increased susceptibility that we observed

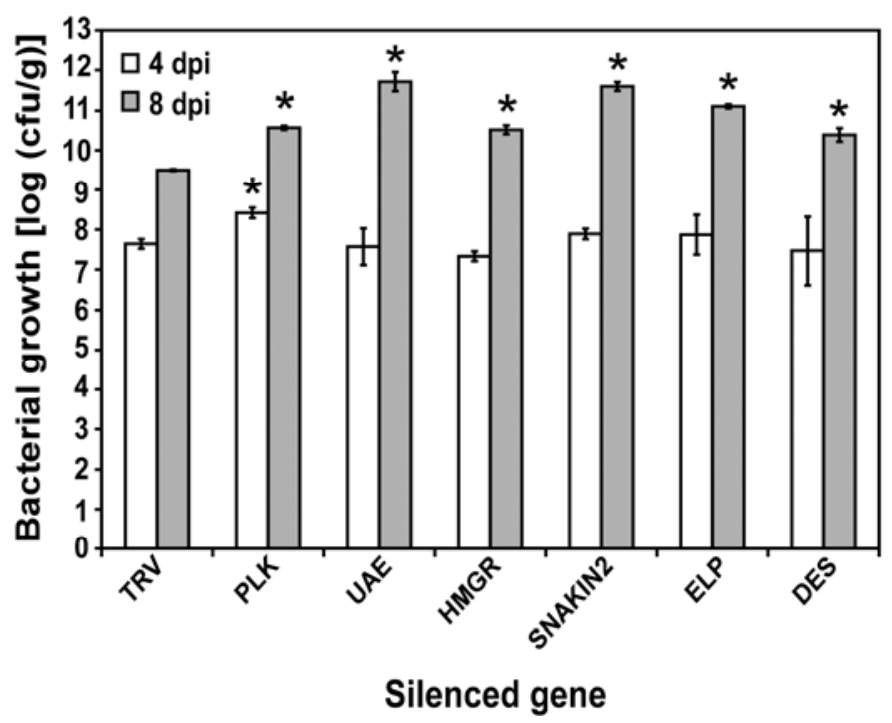

Fig. 4. Growth of Clavibacter michiganensis subsp. michiganensis in silenced stem tissue of Nicotiana benthamiana plants. Two weeks after silencing, plants were inoculated with a suspension of $C$. michiganensis subsp. michiganensis at $10^{8} \mathrm{CFU} / \mathrm{ml}$ into the stem at the third leaf from the bottom and bacterial populations were estimated in stems of the silenced and nonsilenced Tobacco rattle virus (TRV)-inoculated control plants harvested at 4 and 8 days postinoculation (dpi). Gene targeted by virus-induced gene silencing is indicated under each pair of bars. Each bar represents the average and standard deviation (SD) derived from sampling three stems from three independently silenced plants (one stem per plant). Data represent the mean \pm SD $(n=3)$ and are representative of two independent experiments. Asterisks denote significant differences $(P<0.05)$ in the bacterial titer in silenced plants compared with TRV-only infected nonsilenced control plants at each time point, according to Student's $t$ test. 
in plants silenced for the DES gene, may indicate a role for DESderived divinyl ethers in combination with other oxylipins in the basal defense reaction to $C$. michiganensis subsp. michiganensis.

$H M G R$-silenced plants inoculated with $C$. michiganensis subsp. michiganensis developed wilt symptoms much earlier and harbored more bacteria when compared with nonsilenced plants, suggesting a role for $H M G R$ in the basal defense to C. michiganensis subsp. michiganensis infection. In potato tubers, both enzyme activity and mRNA levels of $H M G R$ were shown to increase in response to pathogen infection and elicitor treatment $(7,8,38,42)$. Direct manipulations of tuber $H M G R$ levels by pretreatment with defense elicitors (arachidonic acid) or specific inhibitors of HMGR (mevinolin) significantly impacted disease responses to the soft-rotting bacterium, E. carotovora (42), suggesting that $H M G R$ regulation plays a key role in mediating disease resistance.

A set of tomato transcription factors-Pti4, Pti5, and Pti6 (Pti:Pto-interacting)—were shown to activate defense responses when expressed in Arabidopsis (23). A Pto-responsive gene and Pti5, in particular, were induced 6- and 163-fold, respectively, in tomato inoculated with $C$. michiganensis subsp. michiganensis (2). Results reported here showed that susceptibility to disease and bacterial growth increased in plants silenced for the PLK gene. However, the bacterial titer increase in $P L K$-silenced plants was smaller when compared with plants silenced for the other five genes. This observation was consistent in both experimental repeats (Fig. 4; Supplementary Figure S2). Despite the smaller increase in $C$. michiganensis subsp. michiganensis titer, the fact that $P L K$-silenced plants showed advanced wilt symptoms indicates that other factors besides bacterial multiplication contribute to disease establishment and severity.

This study has identified six genes that play a significant role in the host basal defense-related reaction to $C$. michiganensis subsp. michiganensis. Utilizing $N$. benthamiana as an experimental host of $C$. michiganensis subsp. michiganensis adds to the growing set of tools that can be used to study this pathogen. In combination with transcript expression profiling experiments, SAGE, and other gene discovery methods, VIGS will be a powerful and effective approach to characterize the host-pathogen interaction and for identifying new target genes linked to plant tolerance or resistance to C. michiganensis subsp. michiganensis.

\section{ACKNOWLEDGMENTS}

We thank H. Lange and J. Oliver (Cornell University) as well as S. Eck and C. Ferris (Hobart and William Smith Colleges) for technical assistance, and S. Ekengren and G.. Martin (Boyce Thompson Institute) for providing the 160 tomato TRV clones used in this study. This research was supported by the President's Council of Cornell Women, the office of the Provost of Hobart and William Smith Colleges, the New York State Agricultural Experiment Station, and the United States-Israel Binational Agricultural Research and Development Fund (award no. IS-4047-07).

\section{LITERATURE CITED}

1. Almasia, N. I., Bazzini, A. A., Hopp, H. E., and Vazquez-Rovere, C. 2008. Overexpression of snakin-1 gene enhances resistance to Rhizoctonia solani and Erwinia carotovora in transgenic potato plants. Mol. Plant Pathol. 9:329-338.

2. Balaji, V., Mayrose, M., Sherf, O., Jacob-Hirsch, J., Eichenlaub, R., Iraki, N., Manulis-Sasson, S., Rechavi, G., Barash, I., and Sessa, G. 2008. Tomato transcriptional changes in response to Clavibacter michiganensis subsp. michiganensis reveal a role for ethylene in disease development. Plant Physiol. 146:1797-1809.

3. Baulcombe, D. C. 1999. Fast forward genetics based on virus-induced gene silencing. Curr. Opin. Plant Biol. 2:109-113.

4. Baulcombe, D. C. 2002. RNA silencing. Curr. Biol. 12:R82-R84.

5. Berrocal-Lobo, M., Segura, A., Moreno, M., Lopez, G., Garcia-Olmedo, F., and Molina, A. 2002. Snakin-2, an antimicrobial peptide from potato whose gene is locally induced by wounding and responds to pathogen infection. Plant Physiol. 128:951-961.
6. Chalupowicz, L., Cohen-Kandli, M., Dror, O., Eichenlaub, R., Gartemann, K.-H., Sessa, G., Barash, I., and Manulis-Sasson, S. 2010. Sequential expression of bacterial virulence and plant defense genes during infection of tomato with Clavibacter michiganensis subsp. michiganensis. Phytopathology 100:252-261.

7. Chappell, J., VonLanken, C., and Vogeli, U. 1991. Elicitor-inducible 3hydroxy-3-methylgUitaryl coenzyme A reductase activity is required for sesquiterpene accumulation in tobacco cell suspension cultures. Plant Physiol. 97:693-698.

8. Choi, D., Ward, B. L., and Bostock, R. M. 1992. Differential induction and suppression of potato 3-hydroxy-3-methylgkitaryl coenzyme A reductase genes in response to Phytophthora infestans and its elicitor arachidonic acid. Plant Cell 4:1333-1344.

9. Chun, W. C. C. 1982. Identification and detection of Corynebacterium michiganense in tomato seed using the enzyme-linked immunosorbent assay. MS thesis, University of Hawaii, Honolulu.

10. Davies, H. A., Daniels, M. J., and Dow, J. M. 1997. Induction of extracellular matrix glycoproteins in Brassica petioles by wounding and in response to Xanthomonas campestris. Mol. Plant-Microbe Interact. 10:812-820.

11. Davis, M. J., Gillespie, J. A., Vidaver, A. K., and Harris, R. W. 1984. Clavibacter: a new genus containing some phytopathogenic coryneform bacteria, including Clavibacter xyli subsp. xyli sp. nov., subsp. nov. and Clavibacter xyli subsp. cynodontis subsp. nov., pathogens that cause ratoon stunting disease of sugarcane and bermudagrass stunting disease. Int. J. Syst. Bacteriol. 34:107-117.

12. Dong, Y., Burch-Smith, T. M., Liu, Y., Mamillapalli, P., and DineshKumar, S. P. 2007. A ligation-independent cloning tobacco rattle virus vector for high-throughput virus-induced gene silencing identifies roles for NbMADS4-1 and -2 in floral development1[W] OA]. Plant Physiol. 145:1161-1170.

13. Dreier, J., Meletzus, D., and Eichenlaub, R. 1997. Characterization of the plasmid encoded virulence region pat- 1 of phytopathogenic Clavibacter michiganensis subsp. michiganensis. Mol. Plant-Microbe Interact. 10:195-206.

14. Ekengren, S. K., Liu, Y., Schiff, M., Dinesh-Kumar, S. P., and Martin, G. B. 2003. Two MAPK cascades, NPR1, and TGA transcription factors play a role in Pto-mediated disease resistance in tomato. Plant J. 36:905-917.

15. Fradin, E. F., Zhang, Z., Ayala, J. C. J., Castroverde, C. D. M., Nazar, R. N., Robb, J., Liu, C. M., and Thomma, P. H. J. 2009. Genetic dissection of Verticillium wilt resistance mediated by tomato Ve $1^{1[\mathrm{C}][\mathrm{W}][\mathrm{OA}]}$. Plant Physiol. 150:320-332.

16. Gartemann, K.-H., Abt, B., Bekel, T., Burger, A., Engemann, J., Flügel, M., Gaigalat, L., Goesmann, A., Gräfen, I., Kalinowski, J., Kaup, O., Kirchner, O., Krause, L., Linke, B., McHardy, A., Meyer, F., Pohle, S., Rückert, C., Schneiker, S., Zellermann, E. M., Pühler, A., Eichenlaub, R., Kaiser, O., and Bartels, D. 2008. The genome sequence of the tomatopathogenic actinomycete Clavibacter michiganensis subsp. michiganensis NCPPB382 reveals a large island involved in pathogenicity. J. Bacteriol. 190:2138-2149.

17. Gartemann, K.-H., Kirchner, O., Engemann, J., Grafen, I., Eichenlaub, R., and Burger, A. 2003. Clavibacter michiganensis subsp. michiganensis: first step in understanding of virulence of a gram-positive phytopathogenic bacterium. J. Biotechnol. 106:179-191.

18. Gleason, M. L., Braun, E. J., Carlton, W. M., and Peterson, R. H. 1991. Survival and dissemination of Clavibacter michiganensis subsp. michiganensis in tomatoes. Phytopathology 81:1519-1523.

19. Göbel, C., Feussner, I., Hamberg, M., and Rosahl, S. 2002. Oxylipin profiling in pathogen-infected potato leaves. Biochem. Biophys. Acta 1584:55-64.

20. Göbel, C., Feussner, I., and Rosahl, S. 2003. Lipid peroxidation during the hypersensitive response in potato in the absence of 9-lipoxygenases. J. Biol. Chem. 278:52834-52840.

21. Göbel, C., Feussner, I., Schmidt, A., Scheel, D., Sanchez-Serrano, J., Hamberg, M., and Rosahl, S. 2001. Oxylipin profiling reveals the preferential stimulation of the 9-lipoxygenase pathway in elicitortreated potato cells. J. Biol. Chem. 276:6267-6273.

22. Goritschnig, S., Zhang, Y., and Li, X. 2007. The ubiquitin pathway is required for innate immunity in Arabidopsis. Plant J. 49:540-551.

23. Gu, Y.-Q., Wildermuth, M. C., Chakravarthy, S., Loh, Y.-T., Yang, C., He, X., Han, Y., and Martin, G. B. 2002. Tomato transcription factors Pti4, Pti5, and Pti6 activate defense responses when expressed in Arabidopsis. Plant Cell 14:817-831.

24. Hauck, P., Thilmony, R., and He, S. Y. 2003. A Pseudomonas syringae type 111 effector suppresses cell wall-based extracellular defense in susceptible Arabidopsis plants. Proc. Natl. Acad. Sci. USA 100:8577-8582.

25. Jackson, A. L., Bartz, S. R., Schelter, J., Kobayashi, S. V., Burchard, J., Mao, M., Li, B., Cavet, G., and Linsley, P. S. 2003. Expression profiling reveals off-target gene regulation by RNAi. Nat. Biotechnol. 21:635-637.

26. Jahr, H., Bahro, R., Burger, A., Ahlemeyer, J., and Eichenlaub, R. 1999. 
Interactions between Clavibacter michiganensis and its host plants. Environ. Microbiol. 1:113-118.

27. Jahr, H., Dreier, J., Meletzus, D., Bahro, R., and Eichenlaub, R. 2000. The endo- $\beta$-1,4-glucanase CelA of Clavibacter michiganensis subsp. michiganensis is a pathogenicity determinant required for induction of bacterial wilt of tomato. Mol. Plant-Microbe Interact. 13:703-714.

28. Kovalskaya, N., and Hammond, R. W. 2009. Expression and functional characterization of the plant antimicrobial snakin-1and defensin recombinant proteins. Protein Exp. Purif. 63:12-17.

29. Liu, Y., Schiff, M., and Dinesh-Kumar, S. P. 2002. Virus-induced gene silencing in tomato. Plant J. 31:777-786.

30. Liu, Y., Schiff, M., Serino, G., Deng, X.-W., and Dinesh-Kumar, S. P. 2002. Role of SCF ubiquitin-ligase and the COP9 signalosome in the $N$ gene-mediated resistance to tobacco mosaic virus. Plant Cell 14:1483-1496.

31. Meletzus, D., Bermpohl, A., Drier, J., and Eichenlaub, R. 1993. Evidence for plasmid-encoded virulence factors in the phytopathogenic bacterium Clavibacter michiganensis subsp. michiganensis NCPPB382. J. Bacteriol. 175:2131-2136.

32. Mysore, K. S., Crasta, O. R., Tuori, R. P., Folkerts, O., Swirsky, P. B., and Martin, G. B. 2002. Comprehensive transcript profiling of Pto- and Prfmediated host defense responses to infection by Pseudomonas syringae pv. tomato. Plant J. 32:299-315.

33. Peart, J. R., Lu, R., Sadanandom, A., Malcuit, I., Moffett, P., Brice, D. C., Schauser, L., Jaggard, D. A. W., Xiao, S., Coleman, M., Dow, M., Jones, J. D. G., Shirasu, K., and Baulcombe, D. C. 2002. Ubiquitin ligaseassociated protein SGT1 is required for host and non-host disease resistance in plants. Proc. Natl. Acad. Sci. USA 99:10865-10869.

34. Ron, M., and Avni, A. 2004. The receptor for the fungal elicitor ethyleneinducing xylanase is a member of a resistance-like gene family in tomato. Plant Cell 16:1604-1615.
35. Rotenberg, D., Thompson, T. S., German, T. L., and Willis, D. K. 2006. Methods for effective real-time RT-PCR analysis of virus-induced gene silencing. J. Virol. Methods 138:49-59.

36. Rustérucci, C., Montillet, J. L., Agnel, J. P., Battesti, C., Alonso, B., Knoll, A., Bessoule, J. J., Etienne, P., Suty, L., Blein, J. P., and Triantaphylidès, C. 1999. Involvement of lipoxygenase-dependent production of fatty acid hydroperoxides in the development of the hypersensitive cell death induced by cryptogein on tobacco leaves. J. Biol. Chem. 274:36446-36455.

37. Segura, A., Moreno, M., Madueno, F., Molina, A., and Garcia-Olmedo, F. 1999. Snakin-1, a peptide from potato that is active against plant pathogens. Mol. Plant-Microbe Interact. 12:16-23.

38. Stermer, B. A., and Boslock, R. M. 1987. Involvement of 3-hydroxy-3methylglutaryl coenzyme A reductase in the regulation of sesquiterpenoid phytoalexin synthesis in potato. Plant Physiol. 84:404-408.

39. Thomas, C. L., Jones, L., Baulcombe, D. C., and Maule, A. J. 2001. Size constraints for targeting post-transcriptional gene silencing and for RNAdirected methylation in Nicotiana benthamiana using Potato virus $X$ vector. Plant J. 25:417-425.

40. Varga, G. J., Szegedi, E., Ott, P. G., and Szabo, E. 2006. Early basal resistance inhibits tumor induction by Agrobacterium tumefaciens. Cereal Res. Commun. 34:97-100.

41. Wei, G., and Shirsat, A. H. 2006. Extensin over-expression in Arabidopsis limits pathogen invasiveness. Mol. Plant Pathol. 7:579-592.

42. Yang, Z., Park, H., Lacy, G. H., and Cramer, C. L. 1991. Differential activation of potato 3-hydroxy-3-methylglutaryl coenzyme A reductase genes by wounding and pathogen challenge. Plant Cell 3:397-405.

43. Zipfel, C., Robatzek, S., Navarro, L., Oakeley, E. J., Jones, J. D., Felix, G., and Boller, T. 2004. Bacterial disease resistance in Arabidopsis through flagellin perception. Nature 428:764-767. 